\title{
EL SENTIDO DE LA PEDAGOGÍA Y LA DIDÁCTICA EN LAS TECNOLOGÍAS
}

\author{
José Ocampo Rodríguez, Rómulo Gallego Badillo \\ Universidad Pedagógica Nacional.
}

\begin{abstract}
This paper the authors must then ask ourselves and reply about the existence of a Pedagogic an Didactic of technology knowledge from an epistemology examination, concerning disciplines structures of such knowledge.
\end{abstract}

\section{RESUMEN}

En este artículo, los autores instan a que nos preguntemos y respondamos a su vez, por la existencia de una pedagogía y una didáctica del conocimiento tecnológico desde un examen epistemológico, relacionado con la estructura de las disciplinas.

\section{INTRODUCCIÓN}

Pensar una pedagogía y una didáctica de las tecnologías es un ejercicio intelectual que los autores, con otros colegas (Gallego Badillo, R. et al., 1986), vienen haciendo desde hace ya varios años. Hay que recordar que desde una visión constructivista-deductivista se planteó que la posibilidad de esa pedagogía y de esa didáctica sólo es factible si se abandonaba el dominante esquema positivista (Comte, A., 1984), en el que la tecnología era una aplicación sistemática de los conocimientos científicos en los sectores de la producción y de los servicios especializados. Para Comte los científicos eran los encargados de descubrir con leyes que rigen el funcionamiento del mundo, mientras que la tecnología se ocupaban de la aplicación de tales leyes. Dentro de esta mirada la didáctica de la tecnología era reducida casi a un simple entrenamiento, se trataba pues de un ejercicio mecánico enclavado en la psicología Behaviorista y mucho más precisamente en el conductismo operante Skinneriano y sus programas instruccionales.

Salir de esta posición significó volver a hacer la pregunta por la naturaleza de los saberes tecnológicos, apoyada ella en una revisión histórica de cómo habían sido construidos, desde Arquímedes, pasando por Tcesibio de Alejandría (los geómetras mecánicos), hasta llegar a Watt, por lo menos. La pesquisa fue enriquecedora, puesto que condujo al convencimiento de que era factible proponer una estructura Epistemológica, del carácter conceptual y metodológico para éste sector de la cultura universal. Desde ahí se vio la necesidad de pluralizar es decir no hablar de tecnología sino de tecnologías, cada una de ellas en sus propias estructuras disciplinarias; además, de que cada una de ellas era una construcción del intelecto de los tecnólogos respectivos, construcción que era eminentemente social, al igual que las ciencias experimentales (Hodson, D., 1988), esto es, que cada saber tecnológico era producto del colectivo de investigadores en su campo, 
efecto para el cual se apoyaban conceptual y metodológicamente en las elaboraciones de los científicos, un apoyo que va mas allá de la simple aplicación (Gallego Badillo, 1995).

Fue interesante encontrar como es Galileo quien, al introducir la Episteme de las matemáticas en el saber y el quehacer de los artesanos, los constructores de máquinas (El torno por ejemplo), muta la técnica en tecnología (Koyre, A., 1979); pero es también Galileo quien crea, al apoyarse en un saber modificado de los artesanos, el concepto de experimente en ciencias, es decir, el espacio instrumental y por lo tanto tecnológico en el que se hace factible la demostración de las hipótesis matemáticas sobre el comportamiento del mundo. Dicha puntualización llegó a la convicción de que las relaciones entre ciencias experimentales y tecnologías no podían plantearse desde la óptica simplista de la aplicación, ya que ambas dependen unas de las otras, sin que quepan reduccionismos.

Se postuló entonces, cómo ya se dijo una estructura epistemológica matriz que sirviera de guía para el análisis de la naturaleza del saber, sus fundamentos, de cada una de las formas de realizarse el ser humano como tecnólogo, estructura ésta conformada por las categorías siguientes: Sistema, como objeto de saber y de investigación; MODELO lógicomecánico, como el cuerpo conceptual y metodológico, desde el cual se da cuenta empleando las matemáticas (ecuaciones diferenciales), descriptiva y explicativamente del correspondiente objeto del saber y de investigación, que tiene razón justamente porque emerge de dicho cuerpo, como problemática de saber (Bachelard, G., 1978); DISEÑO, entendido cómo esa función que transforma hipótesis abstractas en concreciones constructivas; el PROTOTIPO que es la concreción misma y que se constituye como el espacio experimental del tecnólogo, esto es, ese propósito de ver funcionando lo que imaginó, dentro de los límites de precisión y exactitud en los que lo imaginó; finalmente, LAS REGLAS DE PRODUCCIÓN, categoría esta adecuada si se atiende a lo dicho de que cada una de las tecnologías son construcciones de los tecnólogos en sus comunidades de especialistas.

Puntualizando lo anterior se hizo indispensable inscribir la propuesta dentro de un ámbito cultural, social, político y económico, ya que era en tal inscripción la que permitía comprender las condiciones externas (Lakatos, 1., 1983), que exigían y apoyaban la construcción de los saberes tecnológicos, no sólo en el sentido de la fabricación de máquinas e instrumentos indispensables por ejemplo, sino que también suministraba los recursos financieros para sostener los grupos y las investigaciones de esos colectivos de tecnólogos. En la perspectiva económica, es claro que tales colectivos son quienes más directamente convierten su saber en mercancía, es decir, en objeto con valor de uso y con valor de cambio; algo que viene siendo así, desde cuando, ad portas de la modernidad, los comerciantes pusieron a producir a los artesanos para personas desconocidas. Se recreó entonces el concepto de mercado y el de cliente, como categorías abstractas a la vez que los artefactos, al adquirir su condición de mercancía, se hicieron igualmente objetos abstractos, después vendrá la revolución industrial que cimentaría definitivamente las transformaciones anotadas. Hoy se habla de megamercado y de tecnofactos.

En la actualidad estas condiciones externas se han vuelto tan dominantes que la mirada epistemológica se ha extendido, hasta considerar que la validez de los saberes científicos y tecnológicos tiene que ser planteada mucho más allá de sus estructuras lógicas internas y de los apoyos empíricos en que se sustentan para considerarlos como verdaderos sistemas de producción. En éste sentido, losen porque desde cada uno de ellos se debe construir más y mejor saber, a la vez que generar mercancías, con sus respectivos valores de uso y valores de cambio, adquiribles en los megamercados. En 
éste caso piénsese como por ejemplo, que la misma información científica o tecnológica se vende y se compra, sobre todo si es altamente competitiva. Por supuesto que aquella que es obsoleta, o que se ha vuelto o obsoleta ha perdido su valor, sus registros de patentes y derechos de autor han finiquitado, quedando únicamente como patrimonio histórico de la humanidad.

Esta nueva óptica ha exigido cambios indispensables en las concepciones y praxis pedagógica y didáctica, por lo menos en el campo de la enseñanza de las ciencias experimentales y de las tecnologías. Dejó de tener sentido aprender para repetir, puesto que ahora hay que hacerlo para crear y producir competitivamente; de ahí que se estén formulando proyectos curriculares que persigan la construcción de ámbitos pedagógicos y didácticos para que los estudiantes y los profesores al mismo tiempo reconstruyan y construyan nuevas competencias cognoscitivas (Gallego Badillo, R., 1999). Además, hay que recalcar en el hecho de que esa enseñanza y ese aprendizaje repetitivo para desempeñarse como operario en un puesto de una fábrica taylorista y Fordiana, está dejando su paso a los servomecanismos, esto es, a los tecnofactos producto de la mecatrónica, todo, porque cada vez es más cierto el principio tecnológico de que "si una actividad humana es planteable en su algoritmo, es factible construir, fabricar la máquina que la haga por un ser humano".

\section{UNA PERSPECTIVA HISTORICA}

Hay que recordar aquí que la Escuela entre Griegos primero y Romanos después fue una institución creada para que educaran los hijos de la Aristocracia. Es esta escuela, en términos latinos, la del trivium y el quadrivium. En ella actuaban los profesores, palabra ésta emparentada con la de profeta, el que tiene el mensaje revelado y por tanto, la verdad: profesor es el que tiene la luz y puede en consecuencia señalar los caminos, indicar los senderos, los senderos correctos: Desde ésta posición pontifica y se ha de pensar y hacer como él lo dice, algo que es indiscutible. En correspondencia ese profesor profeta tiene alumnos, palabra esta que viene de a-luminae, el que carece de luz y es consciente de su situación, por lo que se ve combinado a buscar a aquel que la posee y, por supuesto, ese profesor-profeta vive en persecución de alumnos.

Las llamadas artes mecánicas, en oposición a las denominadas artes nobles, las que se recogían en el trivium y el quadrivium, eran aquellas para cuyo aprendizaje no se requería del concurso del intelecto y eran esas artes mecánicas las ejercidas por los esclavos o los extranjeros o ciudadanos. Se creía que tales artes innobles, como también se les catalogaba, eran accesibles por la simple observación e imitación. Se dice que es el Emperador NUMA quien organiza por primera vez a los artesanos en corporaciones, dándoles un estatuto de legalidad para el ejercicio correspondiente. Surgen así, para dar tan sólo un ejemplo, las corporaciones de los Caldereros, de los plateros, de los peleteros, de los carpinteros y así, sucesivamente, con el transcurrir del tiempo en el seno de estas organizaciones que van a surgir los Ilamados Collegia-artificum, el sistema de formación de los artesanos; dígase de paso que son los colegios, las instituciones educativas, históricamente hablando de formación en las artes. Es también en su interior que se crear la categoría de maestro, palabra ésta que se deriva del latín STRUERE que significa construir, de ahí que piense que el maestro sea el constructor mayor, el más avezado el que domina su arte y ha demostrado públicamente con sus obras ese dominio. El maestro tiene discípulos, no alumnos. Los colegios de los artesanos merecen una revaloración histórica, puesto que es en ellos en los que se produce esa transformación conceptual y 
metodológica que va a conducir a la formación de los constructores de máquinas, saber éste con el cual se va a encontrar Galileo, relojero y pulidor de lentes.

Haciendo los saltos históricos necesarios, hay que decir que en lo educativo se produce una gran revolución con las propuestas de Martín Lutero, personaje éste que transformó las ideas de Erasmo de Rotterdan, en un proyecto religioso nuevo, a la vez que cultural y político, se está en el siglo XVI y no en el XVII que es el de Galileo. Lutero en su obra "Manifiesto a la nobleza Cristiana de Alemania" dice en uno de sus planteamientos que el único juez de la doctrina es el pueblo y, por tanto, el encargado de interpretar la escritura. Se sobreentiende que para que ese pueblo sea el intérprete de la Escritura tiene que necesariamente saber leer y escribir, en esa función interpretativa, que se aparta radicalmente de cualquier noción que aluda al repetir al pie de la letra, para tal efecto, se hará necesario crear la escuela y la educación pública para todos, es en éste contexto que, J. Calvino, partidario de la REFORMA, escribe su libro "Instituciones Cristianas"; libro en el cual, por primera vez aparece en occidente registrada la palabra PEDAGOGÍA, ligada a la Educación, en términos de una educación para todos, como derecho fundamental: La pedagogía desde lo político es democrático y tiene una fundamentación en la democracia, por lo que se enclava en lo laico, en lo civil, y en la libertad.

Ya, a mediados del siglo XVIII y vislumbrado el nacimiento del XIX se están creando las condiciones culturales, sociales, políticas y económicas que van a producir lo que históricamente se conoce como la primera REVOLUCIÓN INDUSTRIAL y, de algunos nuevos conceptos de educación y escuelas públicas se están haciendo cada vez más reales en sus praxis, como también el derecho a la educación. Dígase que se está en un período de tránsito, en el que se está de la misma manera creando una nueva estructura social y es la exigencia que ella demanda la que le piden a la educación que formule los procesos a través de los cuales se haga factible dicho tránsito. Es aquí en donde Juan Amos Comenio le dice a occidente que él tiene la fórmula, a la cual le da el nombre de DIDACTICA. En su obra. Didáctica Magna, establece que ella, la didáctica, es el arte de enseñarle todo a todos con ahorro de tiempo y fatiga.

Hay que entenderlo, eso de enseñarle todo a todos sólo es explicable en el interior de una concepción democrática y una praxis de la democracia. Los sabores construidos por la humanidad, como patrimonio de ella tienen que ser accesibles a todo el mundo sin distingo de credo, raza, o posición social. Por otro lado, un examen juicioso y sistemático de la afirmación de que la didáctica es un arte, solo es comprensible en la perspectiva de la transmutación que Galileo hace de la técnica en tecnología, aún cuando hay que decirlo, Comenio, aparentemente, poco comprendió esa revolución que Galileo había hecho. En consecuencia podrá pensarse que aquello a lo que aspira Comenio es que su didáctica tenga el mismo estatuto epistemológico del pensar y del proceder con arreglo a la manera cómo los tecnólogos en esa época, pensaban y resolvían los problemas. La Didáctica es hija de la modernidad.

Más allá de lo establecido y forzando el discurso habría que pensar (Labastidas, J., 1979) en que la propuesta didáctica tiende a resolver un problema, y ya en la primera revolución industrial, como es el de convertir campesinos analfabetas en obreros letrados, obreros de las fábricas. Como se trata de un cambio de concepción y de proyecto ético de vida, ese trabajo didáctico tiene que ser con ahorro de tiempo y fatiga, es decir, no puede ser un proceso traumático. Entiéndase que se está en un cambio del sistema de producción, esto es, del propio de la sociedad feudal al de la fábrica, centrado ésta última en los sabores científicos y tecnológicos. Para producir dentro de esa sociedad feudal que 
es eminentemente repetitiva no se requiere de saber leer y escribir, ya que el hijo del campesino, dentro de tal sistema sólo le basta con imitar, repetir y seguir al pie de la letra las instrucciones orales dadas por su padre. En la sociedad fabril hay que saber leer y escribir, en términos de interpretación, las informaciones requeridas para desempeñarse eficientemente en ese sistema de producción. Por eso, la nueva sociedad que está emergiendo crea y defiende el derecho a la educación, a la vez que estipula el deber, so pena de sanciones, de que los padres tienen que enviar sus hijos a la Escuela. Sólo en la sociedad que hicimos el tránsito del sistema de producción feudal al de producción fabril fueron aquellos que derrotaron el analfabetismo.

A los autores del presente artículo se les antojó afirmar que la construcción de la Pedagogía y de la Didáctica no es ajena a la modernidad y a la emergencia de la que se va a llamar las sociedades científicas y tecnológicas, en consecuencia y desde éstos parámetros tiene sentido el que se vuelva a preguntar por una pedagogía y una didáctica de las tecnologías; mucho más allá y enclavados en las relaciones del mundo actual, de eso que se ha llamado la sociedad del conocimiento, es indispensable pensar en un nuevo derecho, como es el de que todos los ciudadanos tengan acceso a una alfabetización básica en ciencias y en tecnologías (Fourés. 1997).

Dentro del contexto de una globalización con sus megamercados, regida por los productos de los saberes científicos y tecnológicos, aún cuando la economía sea hoy el paradigma desde el cual se está determinando, una alfabetización científica y tecnológica básica que des-borda, como ya se ha dicho la repetición al pie de la letra, con miras a que los ciudadanos y las ciudadanas puedan participar en la toma de decisiones, acerca de los proyectos estatales o de las empresas privadas que tienen que ver con el desarrollo científico y tecnológico. Pero, al mismo tiempo que construyan las suficientes estructuras conceptuales y metodologías para decidir que han de comprar y porqué razones adquirir en esos megamercados, tecnofactos, que como mercancías son productos de esos sabores científicos y tecnológicos; es decir, tomar distancia en relación la publicidad que intenta que se sea usuario de aquello que lógicamente no necesita.

\section{DE LO PEDAGÓGICO Y DE LO DIDÁCTICO}

Pensando en lo pedagógico hay que decir de entrada, que hay muchas discusiones al respecto de aquello que se ocupa la pedagogía. Desde la idea de que hace relación a lo educativo, y, por tanto a la educabilidad (Gallego Badillo, R., y Pérez Miranda, R., 1999), en el terreno objeto del presente artículo se hace necesario preguntar y discutir qué es aquello que se puede entender por una educación tecnológica indispensable para todos los ciudadanos y ciudadanas, en el mundo actual. Claro que esta pregunta conduce también a interrogarse por aquello que se comprende con la expresión CULTURA TECNOLÓGICA. Es así, por cuanto todo proceso educativo, tanto en sus perspectivas generales como particulares, concretándose éstos últimos en proyectos curriculares específicos se realiza en el interior de un proyecto cultural, social, político y económico característico. Por supuesto, que preguntar por una Educación tecnológica en el seno de una cultura tecnológica implica necesariamente interrogarse por aquello que en cada época histórica y desde las elaboraciones de las comunidades de tecnólogos se entiende por tecnología, intrínsecamente; contando con que desde los especialistas en Antropología cultural, en sociología del conocimiento, en politología y en economía tienen sus propias versiones externa de las razones y sentidos de la necesidad de una Educación en tecnología. 
En este mismo sentido, pensar en una Pedagogía de los saberes tecnológicos sería aquel que intenta develar las razones por las cuales éstos saberes se constituyen en un campo de formación, tanto desde la perspectiva de la generalidad, de la alfabetización tecnológica, como de la decisión personal de hacerse tecnólogo en una de las estructuras conceptuales y metodológicas que determinan la constitución de cada saber tecnológico.

Esto es, de esa decisión de pertenecer a un colectivo de especialistas que tiene como horizonte de sentido el producir en su campo más y mejor saber tecnológico.

Enclavado el análisis en torno a la Educabilidad habría que preguntarse, de la misma manera, de las razones por las cuales educarse en una tecnología significa introducirse autónomamente en un esquema de comprensión y de actuación en el mundo, regido por unas reglas comunitarias y unas normas de pensar y de proceder que hacen de aquél, quién las acepta críticamente sabe y es reconocido como perteneciente. Hacerse tecnólogo es generar un sentido de pertenencia a un colectivo específico de especialistas. Esa construcción del sentido de pertenencia es la que llama a cada quien a la participación activa de aquellas formas de organización comunitaria, como simposios, congresos, seminarios y otros del mismo orden en las que se concita a los miembros de la comunidad a discutir los fundamentos y las razones del saber tecnológico, que como sistemas de producción están comprometidos. En este mismo orden de ideas, y para reiterarlo, se trata de un comprometerse con la producción de más y mejor saber en su campo, demostrables en los prototipos que elaboran y ponen en circulación el beneficio de todos los ciudadanos y ciudadanas.

Desde esta perspectiva teórica habría que pensar que el problema de las pedagogías de las tecnologías, tendría que ver con aquellas reflexiones que tienden a dar cuenta de las razones y de los procesos de cómo, cada sociedad pasa de una cultura no tecnológica a la propia, por lo que, de manera rigurosa indagaría de cuáles son los obstáculos de índole epistemológico (Bachelard, G., 1981), como culturales, sociales, políticos y económicos que estarían en la base de la oposición, la convertibilidad, de eso que se llama el pasar de una cultura no tecnológica a la propia. Igualmente y para insistir, en el campo de quien decide hacerse tecnólogos, qué es aquello, con su raigambre, de nuevas culturas, social, política y económica, y, específicamente epistemológica que se opone en su introducción de manera completa en un saber tecnológico determinado para hacerse productor de tecnología en él. De hecho esta reflexión se aparta de aquello que ha sido llamado con "relación de externalidad" en la que se aprende de una forma repetitiva cualquier saber tecnológico como un paquete "téngalo y úselo" porque mañana habrá otro. Es esto el contexto teórico en el que hay que comprender, y delimitar aquello de que, los saberes tecnológicos son aplicaciones para saltar a la idea de que cada modelo lógico-mecánico como objeto de enseñanza no es más que una oportunidad para adentrarse en él con miras a producir tecnología. Quién no se sumerge en las razones de ser, de la forma como se ha producido, jamás será tecnólogo; a lo menos usuario, en decir operario.

En cuanto a la Didáctica de las tecnologías hay que de nuevo, formular las preguntas que le dan constitución, esto pone en tela de juicio concepciones arraigadas que hablan a favor ¿de qué? Basta con dominar un saber tecnológico, cualesquiera, sea él para enseñarle; algo con lo cual carecería de justificación el ocuparse tanto de las reflexiones rigurosas de las didácticas como de las pedagogías; en éste orden de ideas no tendría justificación epistemológica, cultural, social, política y económica, financiar en instituciones ocupadas precisamente de éstos campos. 
Viene aquí el interrogante necesario de que es aquello que se va a entender como proyecto de investigación de lo que significa hablar de las didácticas de las tecnologías. Reduce ella, esa pregunta compleja a unos métodos de cómo enseñar las estructuras disciplinarias que caracteriza cada uno de los sabores tecnológico, o, por el contrario pensar en una didácticas de las tecnologías significa el introducirse en una concepción conceptual y metodológica, en la que el horizonte de sentido es como generar proyectos curriculares para acrecentar el número de hombres y mujeres dedicadas a producir tecnologías. Centrado en el maestro y vale aquí recordar que ese fue quien nació en los colegios de artesanos aquel que rigurosa y sistemáticamente construye las versiones didácticas del saber tecnológico en la que es Maestro, para introducir de manera paulatina a sus alumnos en esa riqueza, como tantas otras, que es el ser Maestro de una tecnología.

Se cree aquí que la pedagogía y la Didáctica de los tecnólogos esta por construirse y que necesariamente ha de partir de hacer une examen tan completo como se quiere de aquello que se entiende por tecnología y de que es ser tecnólogo. De hecho y en lo que toca al departamento de tecnología de la Facultad de Ciencia y Tecnología de la Universidad Pedagógica Nacional es un problema a elaborar en lo tocante a la pedagogía y a la Didáctica de éste sector de la cultura universal. Los autores precisan y debe subrayarse que no basta con ser tecnólogo en un campo disciplinarios específico para ser pedagogo y didáctico del mismo.

Hay que precisar que detrás de la enseñanza de una tecnología hay un problema que no puede ser despachado con cualquier respuesta. Es ese que indaga por la enseñabilidad de cada saber tecnológico, enseñabilidad ésta que como pregunta Epistemológica no puede ser resuelta, desde la misma estructura disciplinar respectiva. Se piensa aquí que las preguntas didácticas pedagógicas en el interior de cada saber tecnológico y en cada momento histórico obliga a aquellos que ejercen como pedagogos y didáctas a construir una versión de su saber que a lo mejor cambia la concepción de los mismos tecnólogos que no ejercen como tecnólogos. Toda construcción didáctica de cada uno de los sabores tecnológicos es una interpretación y por lo tanto una propuesta innovadora que replantea aquello que se concibe como tal.

\section{CONCLUSIONES}

Se piensa en éste artículo que se ha abierto una discusión; con los fundamentos históricos requeridos, para que colectivamente, en el interior de aquellos que están preocupados por el problema de la pedagogía y la didáctica de la tecnología; una conclusión que necesariamente serán producto de ese colectivo y que es necesario animar, siempre y cuando se piense en términos de formar a los nuevos educadores en los sabores tecnológicos. Ya antes se había intentado una reflexión en torno a la pedagogía y la didáctica de estos sabores (Ocampo Rodríguez; y Gallego Badillo R., 1989) en el cual y a partir del cual los autores estaban esperando una discusión crítica de lo que se propone en ese artículo, no obstante no se produjo. 


\section{BIBLIOGRAFÍA}

Bachelard, Gastón. El racionalismo aplicado. Buenos Aires: Editorial Paidós. 1978.

Bachelard, Gastón. La formación del espíritu científico. 9a. Edición. México: Siglo XXI. Edit. 1981.

Comte. A. Curso de filosofía positiva. Lecciones I y II. Barcelona: Editorial Orbis. 1984.

Foures, G. Alfabetización científica y tecnológica, acerca de la finalidad de la enseñanza. Buenos Aires: Ediciones Colihue. 1997.

Gallego Badillo, R.; Ocampo, José. Diseño y evaluación de las estrategias y metodología para la formación científica y tecnológica. U.P.N. CIUP: 1986.

Gallego Badillo, R. Competencia cognitivas. Editorial Magisterio. 1999.

Gallego Badillo, R.; Pérez Miranda, R. Aprendibilidad, educabilidad, enseñabilidad. Revista Colombiana de Educación. 1999.

Hodson, D. Toward a philosophically in science education. Science Education. 1988.

Koyre, A. El mundo del más a menos, al universo de la precisión. Revista Naturaleza. 1979.

Labastidas, Jaime. Producción ciencia y sociedad: Descartes a Marx. 8a. Edición, México: Siglo XXI. 1979.

Lakatus, I. La metodología de los programas de investigación científica. Madrid: Alianza Editorial. 1983.

Ocampo, José; Gallego Badillo, R., Pérez Miranda, H: El salto tecnológico. Revista Actualidad Educativa. 1995. 